\title{
Interdependence of body mass index with handgrip strength and endurance among apparently healthy teenagers
}

\author{
Adebisi I. Hammed, Chigozie O. Obaseki \\ Department of Physiotherapy, University of Benin Teaching Hospital, Benin-City, Edo State, Nigeria.
}

\begin{abstract}
This study investigated the relationship of body mass index (BMI) with handgrip strength and endurance among apparently healthy Nigerian Teenagers. A total of 200 secondary school students participated in this study. Body height and body weight were measured with a wallmounted stadiometer in meters $(\mathrm{m})$ and bathroom weighing scale in kilogram $(\mathrm{kg})$ respectively. Body mass index (BMI) was then calculated using the formula weight $(\mathrm{kg}) /$ height $\left(\mathrm{m}^{2}\right)$. An electronic handgrip dynamometer was used to measure handgrip strength (HGS) in $\mathrm{kg}$ and handgrip endurance (HGE) in seconds (s). The relationship of BMI with HGS and HGE was analyzed using Pearson's product moment coefficient of correlation. Then, differences in BMI, HGS and HGE across gender categories were analyzed using the independent sample t-test. The outcome of this study showed that BMI correlated proportionately and significantly ( $\mathrm{p}<0.05)$ with HGS and HGE. Also, a significant difference $(p<0.05)$ exists in HGS and HGE across gender characteristics. This study therefore concluded that BMI is a determinant of HGS and HGE among apparently healthy Nigerian Teenagers and thus, could be considered as a marker of nutritional and health status, as well as physical fitness of these individuals. Also, a gender difference does influence HGS and HGE among apparently healthy Nigerian Teenagers.
\end{abstract}

Keywords. Anthropometric properties, handgrip endurance, handgrip strength, teenagers.

\section{Introduction}

Y oung population is the back bone of a nation only because they are usually presented with better activity level and lifestyle characteristics. In the daily life of humans, the major proportion of usage of hand involves the dominant hand. Many activities of daily living and sports performance require high activity levels of muscles of the hands and forearms. These muscles are required for execution of some skills involving physical demands and endurance. Recently, anthropometric measures constitute the new vital signs of the 21st century, and therefore warrant urgent assessment if the tide of obesity is to be turned. This is because body mass index (BMI) below 18.5 and over 24.9 is associated with health risk-the latter with hypertension, dyslipidemia, type 2 diabetes, coronary heart disease, stroke, gall bladder disease, osteoarthritis, sleep apnea, respiratory problems, and other systemic conditions. Moreover, the economic and social consequences of these conditions could be overwhelming especially in underdeveloped and developing countries (World Health Organization, 2008). An individual's BMI can be categorized into underweight, normal weight, overweight and obese. Muscular power can be negatively affected in overweight or obese individuals, as a result of both sedentary lifestyle and high physical inactivity. Poor muscle strength has been found to be associated with low body weight and poor nutritional status, and many previous studies have attempted to correlate HGS with anthropometrics to predict the outcome of the former (Mandahawi et al., 2008). Furthermore, the amount of fat-free muscle has been highly correlated with grip strength in teenagers (Henneberg et al., 2001). Increasing levels of testosterone and greater level of sport performance have been observed to be related to increasing grip 
strength in boys entering puberty. These findings could help to explain the increasing grip strength difference between boys and girls throughout the teenage years (Neu et al., 2002).

Handedness is not a discrete variable (right or left), but a continuous one, that can be expressed at levels between strong left and strong right. It is the dominance of one hand over the other, or the unequal distribution between the left and right hands. Handgrip strength (HGS) has long been thought of as a possible predictor of overall body strength (Smith et al., 2006). HGS is an important variable that is physiologically affected by several factors including age, sex, handedness, body height, body weight and BMI among others (Baskaran et al., 2010). In addition, HGS is a form of contraction test that is clinically reliable and mostly measured isometrically to assess the levels of physical fitness and nutrition of an individual. It is commonly used to assess and measure the upper limb muscular strength in all populations (Schlussel et al., 2008). Meanwhile, a decline in HGS can eventually result into diminished functions that is, a reduction in basic and instrumental activities of daily living thereby diminishing health related quality of life of an individual (Savas et al., 2007). This is because hand functionality is considered to be vital in most of the activities involving the upper limb, be it writing, eating, carrying loads, lifting objects, opening or closing doors etc. On the other hand, handgrip endurance (HGE) is the capability of a muscle to maintain a force produced, for lengthy periods, during physical activity. It is not independent of the strength of a brief maximum effort (Edwards et al., 1977). Muscular endurance depends largely on the availability of nutrition for the muscle (Smrithi et al., 2012). Likewise, factors such as cardiorespiratory fitness, skeletal muscle function, blood flow and temperature of the muscle can also affect muscular endurance (Das et al., 2015). Along with basic and instrumental activities of daily living, HGS is essential to many sports such as rock climbing, wrestling, handball, tennis, swimming and so on, and it is thus regarded as a factor in maximizing performance and control (Vassilis, 2012).

Several factors including gender, age, body height, body weight, BMI and handedness have been observed to correlate with grip strength performance (Deepak et al., 2014; Liao, 2014). Visnapuu et al. (2007) also found that body weight and stature are primary indicators of human growth, and concluded that there are significant associations between dominant handgrip strength and general anthropometrics in different age categories. Moreover, Das et al. (2015) reported a significant direct relationship between BMI and handgrip strength and endurance in different weight groups among apparently healthy individuals. Meanwhile, Helmersson et al. (2004) claimed that gender is the most important determining factor of hand grip strength in human. Muscle weakness has been related to some anthropometric parameters such as BMI and body weight (Bohannon, 2001) in adult populations. In effect, many HGS studies in young healthy individuals have shown that anthropometrics such as body height, body weight, BMI, and grip span are directly correlated with HGS (Vaz et al., 2002). Also, a parallel increase of HGS has been demonstrated with age that seems to be highly dependent on the increase in BMI. Infact this could simply reflect sexual dimorphism, due to the action of sex steroid hormones. It has also been reported that physical performance has a strong association with body strength, shape, size, form and structure of an individual (Foo, 2007). In 2014, Bansode et al. found a significant positive relationship of dominant HGS with age, body height, body weight, BMI and hand span of dominant hand in adult males and females. Furthermore, it has been claimed that once males enter the teenage years, they are significantly stronger than their age-matched female counterparts (Hager-Ross et al., 2002). Equally, Lee et al. (2012) submitted that HGS is mainly influenced by age and height in both men and women, and also by BMI in men only among Koreans. In addition, Gunther et al. (2008) stated that among anthropometric variables, grip strength was correlated best with height in both gender, and weight was associated in men, but not in women among Caucasians. In contrast, Silventoinen et al. (2008) established a direct relationship between HGS and height, weight, and BMI in both sexes in a study among Swedish population.

Substantial evidence has pointed to the obvious relationship of BMI with HGS in young individuals but with little evidence of such relationship with HGE. Also, it is worth noting that categories of BMI that are said to associate with HGS and HGE are never the same across communities in the same country and 
neither are they the same across countries. For this reason, the interdependence of BMI with HGS and HGE may not be the same as found elsewhere. This study was therefore carried out to evaluate the association of BMI with HGS and HGE among apparently healthy Nigerian Teenagers.

\section{Methods}

\section{Research design}

This study was a correlational survey of the relationship of BMI with HGS and HGE among apparently healthy Nigerian Teenagers.

\section{Population}

The population for this study included Oyo state school students between the biological ages of 13 and 19 years from three secondary schools in the 2015/2016 academic session.

\section{Sample size and sampling technique}

A total of one hundred (100) male and one hundred (100) female secondary school students participated in this study. They were recruited using proportionate random sampling technique. However, participants with any joint problems of hand, wrist and elbow, history of fracture, neurological disorder, and deformities of upper limb were excluded from the study.

\section{Measurements and data collection}

The ethical approval was obtained from Research Ethics Committee of University of Benin for permission to conduct this study and the participants were then recruited consecutively. HGS was measured using a Camry Electronic Hand Dynamometer (Model: EH101). It comes with dual scale readout of forces in kilograms and pounds. However all readings were recorded in kilograms in the present study. To standardize the test, the following guidelines were established by two evaluators; the arm positioning followed the American Society of Hand Therapists guidelines (Fess, 1992), with the participant comfortably seated with the shoulder slightly forward and the elbow flexed at a $90^{\circ}$ angle. The forearm and wrist were in a neutral position. Three maximum efforts were performed for dominant hand, with threesecond contractions and ten-second rest periods between the attempts. The best of three attempts was recorded by the evaluators. The following test instructions were provided: "you must squeeze the handle as hard as possible keeping both your body and arm in position". The same tone was used during the briefings, and no verbal encouragement was offered. The instrument calibration was assessed periodically throughout the study.

HGE was also determined using a Camry Electronic Hand Dynamometer (Model: EH101). Participants were made to maintain same position as in HGS measurement. HGE test consisted of twelve maximal isometric gripping of dominant hand. Contractions were held for three-seconds, with a five-second rest period between each repetition. Endurance was assessed using the percentage fatigue index value which was calculated by $1^{\text {st }}$ peak torque (PT) minus the last $\left(12^{\text {th }}\right)$ PT, divided by the $1^{\text {st }}$ PT multiplied by 100 . However, the hand that is preferred for daily activities such as writing and eating was taken as the dominant hand.

The standing heights were measured using a stadiometer. Each participant was assessed while in good standing posture on the foot resting on the device with minimal clothing without shoes but with the head facing forward in front font position. The participants also had the shoulders relaxed, arms hanging loosely on both sides, palms facing forward, feet together and knees straight. The height for each participant was taken when the movable headboard was lowered to touch the vertex of the head. The measurements were taken to the nearest 0.1 centimeter.

The calibrated weighing scale was used to measure body weight and was checked for zero balance before each use. Participants were instructed to empty their pockets and remove shoes and any apparel that could interfere with weight measurements. They then stood on the scale looking straight ahead, relaxed and remained motionless without leaning on any object or the wall. Weight measurements were taken when the scale stabilized and recorded to the nearest $0.1 \mathrm{~kg}$ while protecting the confidentiality of the participants' values. BMI was then calculated using the formula weight $(\mathrm{kg}) /$ height $\left(\mathrm{m}^{2}\right)$. 


\section{Data analysis}

The data obtained from this study were analyzed using descriptive and inferential statistics. Anthropometric profile of the participants was summarized using mean and standard deviation. Relationships of BMI with HGS and HGE were analyzed using Pearson's product moment coefficient of correlation, and then differences in BMI, HGS and HGE across gender categories were analyzed using the independent sample t-test. All inferential analyses were performed at the 0.05 alpha levels using the Statistical Package for the Social Sciences (SPSS version 20.0).

\section{Results}

The results are presented in Tables 1-4. The mean and standard deviation values for the BMI, HGS and HGE in the general sample are $21.10 \pm 3.80 \mathrm{~kg} / \mathrm{m}^{2}, 17.07 \pm 4.66$ $\mathrm{kg}$ and 32.60 $\pm 5.89 \mathrm{sec}$ respectively as shown in Table 1 . The descriptive statistics in both male and female samples in each case showing the mean, standard deviation and standard error mean values for each variable are presented and graphically represented in Table 2 and Figure 1 respectively. The relationship of BMI with HGS and HGE is showed in Table 3. Also, an independent t-test for differences in BMI, HGS and HGE across gender categories is presented in Table 4.

This study showed that BMI is a proportionate and significant $(p<0.05)$ correlate of HGS $(\mathrm{r}=.773)$ and HGE $(\mathrm{r}=.642)$. This implies that BMI is a predictor of HGS and HGE among apparently healthy Nigerian Teenagers. It was observed from this study that a significant difference $(p<0.05)$ exists in HGS and HGE across gender characteristics.

\section{Table 1}

Descriptive statistics for general samples $(n=200)$.

\begin{tabular}{lcccc}
\hline & Minimum & Maximum & Mean & SD \\
\hline BMI & 15.93 & 30.20 & 21.10 & 3.80 \\
HGS & 8.90 & 26.60 & 17.07 & 4.66 \\
HE & 18.30 & 47.40 & 32.60 & 5.89 \\
\hline
\end{tabular}

$\mathrm{N}=$ Total number, $\mathrm{BMI}=$ Body Mass Index, HGS= Hand Grip Strength, HGE $=$ Hand Grip Endurance.
Table 2

Descriptive statistics for male and female samples.

\begin{tabular}{lllll}
\hline & Gender & Mean & SD & SE \\
\hline \multirow{2}{*}{ BMI } & Male & 22.789 & 3.88 & .388 \\
& Female & 19.40 & 2.84 & .284 \\
\multirow{2}{*}{ HGS } & Male & 20.76 & 2.95 & .295 \\
& Female & 13.38 & 2.71 & .271 \\
\multirow{2}{*}{ HE } & Male & 36.14 & 4.32 & .432 \\
& Female & 29.06 & 5.08 & .508 \\
\hline
\end{tabular}

BMI = Body Mass Index, HGS= Hand Grip Strength, HGE = Hand Grip Endurance.

Table 3

Correlations of participants' BMI with HGS and HGE for general samples.

\begin{tabular}{lcccc}
\hline & Gender & BMI & HGS & HGE \\
\hline Gender & 1 & & & \\
\multirow{3}{*}{ BMI } & & & & \\
& $-.447^{* *}$ & 1 & & \\
& .000 & & & \\
HGS & 200 & 200 & & \\
& $-.795^{* *}$ & $.773^{* *}$ & 1 & \\
& .000 & .000 & & \\
HE & 200 & 200 & 200 & \\
& $-.602^{* *}$ & $.642^{* *}$ & $.823^{* *}$ & 1 \\
& .000 & .000 & .000 & \\
& 200 & 200 & 200 & 200 \\
\hline
\end{tabular}

BMI = Body Mass Index, HGS= Hand Grip Strength, HGE = Hand Grip Endurance.

Table 4

Independent sample t-test for differences in BMI, HGS and HGE across gender categories.

\begin{tabular}{|c|c|c|c|c|c|}
\hline & F & Sig. & $\mathrm{t}$ & $\mathrm{df}$ & $\mathrm{p}$ \\
\hline \multirow[t]{2}{*}{ BMI } & 26.349 & .000 & 7.028 & 198 & .000 \\
\hline & & & 7.028 & 181.385 & .000 \\
\hline \multirow[t]{2}{*}{ HGS } & 4.269 & .040 & 18.435 & 198 & .000 \\
\hline & & & 18.435 & 196.661 & .000 \\
\hline \multirow[t]{2}{*}{$\mathrm{HE}$} & 2.933 & .088 & 10.607 & 198 & .000 \\
\hline & & & 10.607 & 192.948 & .000 \\
\hline
\end{tabular}




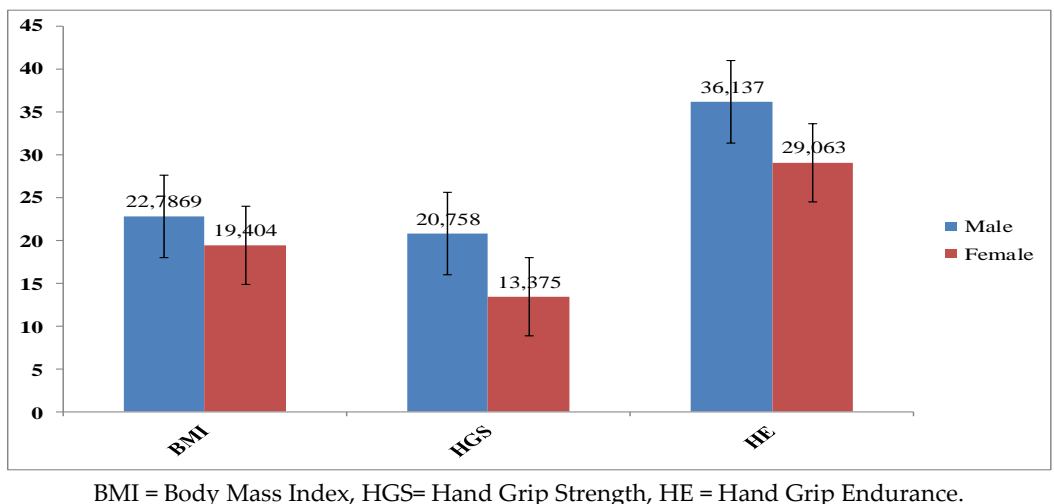

Figure 1. Graphical representation of HGS $(\mathrm{kg})$ and HGE (sec) for male and female samples.

\section{Discussion}

This study suggests that BMI is a significant positive correlate of HGS and HGE. The implication of this is that higher BMI would result to better HGS and HGE. This might be as a result of higher percentage of skeletal muscle mass but not percentage fat mass which can largely be responsible for body heaviness thereby resulting into better or higher HGS and HGE. This finding is in agreement with the findings of Baskaram et al. (2010), Deepak et al. (2014), Liao, (2014), Visnapuu et al. (2007) who reported a positive and significant relationship between BMI and HGS only. Likewise, a very important study by Das et al. (2015) revealed a proportionate and significant relationship of BMI with HGS and HGE among young healthy adults. Therefore, this agreement could mean that higher BMI as a result of higher percentage of skeletal muscle mass is physiologically responsible for total body strength and endurance in human. This by implication means that having higher BMI could be an edge in the proper execution of some skills requiring the use of the hand and involving physical demands and endurance.

Furthermore, males showed a significantly higher HGS and HGE than their female counterparts. The significant difference between gender characteristics as regards HGS and HGE suggests that gender is associated with HGS and HGE among Nigerian school Teenagers. Also, the variation in percentage of skeletal muscle mass development between boys and girls could be responsible for total body strength and by implication affect the execution of some skills requiring the use of the hand and involving physical demands.
However, this could simply reflect sexual dimorphism, due to the action of sex steroid hormones. This might not be a surprise because males are generally seen to be stronger than females in general population. The implication of this finding could be viewed in two different perspectives. Firstly, male gender as the root of initial episode of higher HGS and HGE as a result of male hormone testosterone which can physiologically help in increasing the muscle size and strength by increasing type 2 muscle fibres (which are fast type of fibres with high glycolytic activity). Secondly, better activity characteristics and lifestyle factors among males might not be unconnected with the better HGS and HGE. However, reduced physical fitness and excessive accumulation of adipose tissue might be responsible for impairment of muscle strength among females. This finding is supported by the findings of Neu et al. (2002) who reported that increasing levels of testosterone and greater participation in sports have been found to be related to increasing grip strength in boys entering puberty. Hager-Ross et al. (2002) also submitted that once males enter the teenage years they are generally significantly stronger than age-matched females. Moreover, Helmersson et al. (2004) concluded that gender is the most important factor acting on the values of HGS in human. Nevertheless, only Das et al. (2015) reported in their study that males have shown higher HGS and HGE than females although among young healthy adults. In contrast, the study of Lee et al. (2012) concluded that HGS is mainly influenced by age and height in both men and women among Koreans. These contrasting findings might be a result of variation in study methodology including subject 
characteristics, differences in measuring instruments of HGS, HGE and so on.

\section{Conclusions}

This study therefore concluded that BMI is a determinant of HGS and HGE among apparently healthy Nigerian Teenagers and thus could be considered as a marker of nutritional and health status, as well as physical fitness of these individuals. Also, a gender difference does influence HGS and HGE among apparently healthy Nigerian Teenagers.

It is expected that finding of this study:

- Might establish a simple model to predict HGS and HGE in dominant hand.

- Might also serve as an objective marker of nutritional and health status as well as physical fitness.

Based on the findings, the following recommendations were made: 1 ) BMI must be taken into consideration in hunting and identification of sport talented Teenagers requiring the use of the hand and, higher physical demands and endurance. 2) HGS and HGE measures should be introduced into sports practice to adjudge proper execution of some skills requiring the use of the hand and involving physical strength.

\section{References}

Bansode DG, Borse LJ. Yadav R. Study of correlation between dominant hand's grip strength and some physical factors in adult males and females. International Journal of Pharma Research and Health Sciences, 2014; 2(4): 316-323.

Baskaran C, Arindam G, Chandan P, Bidhan C. Anthropometric traits predict hand grip strength in healthy normal. Journal of Hand \& Microsurgery, 2010; 2(2): 58 - 61.

Bohannon RW. Dynamometer measurements of handgrip strength predict multiple outcomes. Percept Motor Skills, 2001; 93(2): 323-328.

Das A, Dutta M. Correlation between body mass index and handgrip strength and handgrip endurance among young healthy adults. Journal of Evidence based Medicine and Healthcare, $2015 ; 2:$ 3995-4001.

Deepak GB, Laxmikant JB, Rasika DY. Study of correlation between dominant hand's grip strength and some physical factors in adult males and females. Int J Pharm Res Health Sci., 2014; 2(4): 316-323.
Edwards RH, Young A, Hosking GP, Jones DA. Human skeletal muscle function: description of tests and normal values. Clin Sci Mol Med, 1977; 52: 283-290.

Fess EE. Grip Strength. American Society of Hand Therapists. 2nd edition, Chicago, 1992.

Foo LH. Influence of body composition, muscle strength, diet and physical activity on total body and forearm bone mass in Chinese adolescent girls. Br J Nutr, 2007; 98(6): 1281-1287.

Gunther CM, Burger A, Rickert M, Schulz CU. Grip strength in healthy Caucasian adults: reference values. J Hand Surg Am, 2008; 33: 558-565.

Hager-Ross C, Rosblad B. Norms for grip strength in children aged 4-16 years. Acta Paediatr, 2002; 91: 617-625.

Helmersson J, Vessby B, Larsson A, Basu S. Association of type 2 diabetes with cyclooxygenase-mediated inflammation and oxidative stress in an elderly population. Circulation, 2004; 109: $1729-1734$.

Henneberg M, Brush G, Harrison GA. Growth of specific muscle strength between 6 and 18 years in contrasting socioeconomic conditions. Am J Phys Anthropol, 2001; 115: 62-70.

Lee JE, Kim KW, Paik N, Jang HC, Chang CB, Baek GH, Gong HS. Evaluation of factors influencing grip strength in elderly Koreans. Journal of Bone Metabolism, 2012; 19(2): 103-110.

Liao KH. Experimental study on gender differences in hands and sequence of force application on grip and hand-grip control. Int J Occup Saf Ergon, 2014; 20(1): 77-90.

Mandahawi N, Imrhan S, Al-Shobaki S, Sarder B. Hand anthropometry survey for the Jordanian population. International Journal of Industrial Ergonomics, 2008; 38(1112): $966-976$.

Neu CM, Rauch F, Rittweger J, Manz F, Schoenau E. Influence of puberty on muscle development at the forearm. Am J Physiol Endocrinol Metab, 2002; 283: 103-107.

Savas S, Koroglu BK, Koyuncuoglu HR, Uzar E, Celik H, Tamer NM. The effects of diabetic related soft tissue hand lesions and the reduced hand strength on functional disability of hand in type 2 diabetic patients. Diabetes Research and Clinical Practice, 2007; 77(1): 77-83.

Schlussel MM, Anjos LA, Kac G. Hand grip strength test and its use in nutritional assessment. Rev Nutr, 2008; 21: 223-225.

Silventoinen K, Magnusson PK, Tynelius P. Heritability of body size and muscle strength in young adulthood: A study of one million Swedish men. Genet Epidemiol, 2008; 32: 341-9.

Smith T, Martin M, Henry R, Weeks S, Bryant A. Grip strength in relation to overall strength and functional capacity in very old and oldest old females. The Haworth Press Inc., 2006.

Smrithi SC, Shibin GP, Nagaraja S. Influence of various anthropometric parameters on handgrip strength and handgrip endurance in young males and females. Int J Biol Med Res, 2012; 3(3): 2153-2157.

Vassilis G. Reliability of handgrip strength test in Basketball Players. Journal of Human Kinetics, 2012; 31: 25-36. 
Vaz M, Hunsberger S, Diffey B. Prediction equations for handgrip strength in healthy male and female subjects encompassing a wide age range. Ann Hum Biol, 2002; 29: 131-141.

Visnapuu M, Jurimae T. Handgrip strength and hand dimensions in young handball and basketball players. J Strength Cond Res, 2007; 21(3): 923-929.
World Health Organization. Now more than ever: The World Health Report, Geneva: World Health Organization, 2008. 